\title{
Les rapports de gestion de la FMH et de I'ISFM font peau neuve
}

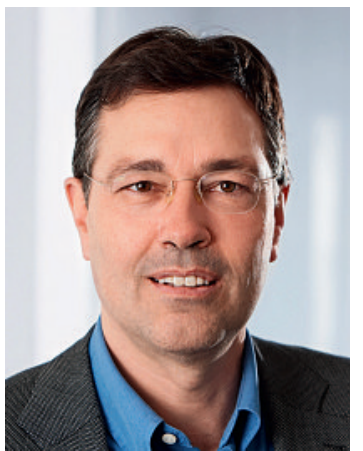

Dans une mise en page entièrement revisitée, le rapport de gestion de la FMH présente désormais les événements de première importance pour le corps médical de manière chronologique. Edité sous la forme d'un magazine, il se veut rajeuni, dynamique et varié. Agréable à lire, il s'est adapté à notre nouveau comportement en matière de lecture.

Le contenu reste inchangé: le rapport de gestion 2010 passe en revue les questions pour lesquelles la FMH s'est engagée au cours de l'année, les résultats obtenus et les moyens utilisés pour y parvenir.

\section{Le rapport de gestion 2010 passe en revue les questions défendues}

\section{par la FMH au cours de l'année.}

Sur le plan politique, en 2010, la FMH a veillé à ce que les médecins puissent continuer d'exercer leur profession dans les meilleures conditions possibles - également du point de vue tarifaire. En tant qu'association professionnelle consciente de ses responsabilités, elle a également défendu des causes fondamentales pour le secteur de la santé, dont le renforcement de la médecine de famille. Comme chaque année, nous avons pris position sur les sujets d'actualité, telles la protection des données, l'alimentation forcée, l'aide au suicide ou encore les nombreuses révisions de lois. Et bien évidemment, nous sommes largement intervenus dans les débats qui dominent le monde politique, au premier plan desquels le Managed care et SwissDRG.

La FMH s'est également montrée très active dans ses projets: nous avons ainsi lancé, conjointement avec $\mathrm{H}+$, notre propre révision du TARMED avec l'objectif d'adapter la structure tarifaire aux réalités actuelles. Dans le domaine SwissDRG, il s'est agi de clore la ronde des propositions dans les délais prévus et de définir les bases devant permettre d'étudier l'impact de l'introduction du tarif, au moyen d'un projet de recherche concomitante.

Afin d'améliorer la visibilité des activités en faveur de la qualité, la FMH - avec le soutien de ses principaux partenaires du secteur de la santé - a réalisé un sondage auprès de quelque 10000 médecins. En 2010, elle s'est en outre associée au prix Swiss Quality Award qui récompense les innovations en matière de gestion de la qualité. Grâce au travail que nous avons accompli, la notoriété de ce prix a sensiblement augmenté.

Le domaine de la formation médicale constitue aussi un volet primordial de notre engagement. Centre de compétences autonome au sein de la FMH, l'Institut suisse pour la formation postgraduée et continue ISFM informe de ses pro-

\section{L'ISFM informe de ses projets dans son} propre rapport de gestion.

jets dans son propre rapport de gestion, afin de s'établir clairement comme l'interlocuteur central pour toutes les questions concernant la formation. Son rapport a également fait peau neuve et se présente dans une mise en page aérée et moderne.

En 2010, l'ISFM a posé les jalons de projets primordiaux, dont le programme de formation postgraduée en médecine interne générale et la plateforme électronique pour la formation continue. Le projet «e-logbook» et l'accréditation sont eux aussi en bonne voie.

A l'instar des années précédentes, le nombre de membres de la FMH a augmenté, dépassant 35 000, soit une augmentation de $1,1 \%$. La FMH est ainsi la plus grande association professionnelle du secteur de la santé. Par ailleurs, la demande pour les titres de spécialistes et les titres de formation approfondie a une fois de plus dépassé nos attentes.

Le résultat financier, dont vous trouverez les détails dans les deux rapports annuels, peut à nouveau être qualifié de solide, ce qu'a également confirmé notre organe de contrôle externe. En d'autres termes, malgré l'augmentation croissante de ses activités, la FMH prouve qu'elle sait gérer les moyens à sa disposition et qu'elle place les intérêts de ses membres au centre de ses préoccupations.

Comme vous pouvez le constater, la FMH, très active, a beaucoup obtenu en 2010 et a su poser des accents importants. En transmettant notre message à l'extérieur, nos rapports de gestion montrent clairement le positionnement fort et durable du corps médical. Cette année à nouveau, tous nos membres recevront ces publications sous la forme conviviale de journaux électroniques, très facilement accessibles en ligne: www.fmh.ch $\rightarrow$ FMH $\rightarrow$ Rapport de gestion, et www. siwf.ch $\rightarrow$ Bases $\rightarrow$ Rapports de gestion.

Bonne lecture! 